DOI https://doi.org/10.30525/978-9934-588-81-5-1.18

\title{
NEW APPROACHES FOR IMPROVING THE EFFECTIVENESS OF THERAPY OF SIMPLE ENDOMETRIAL HYPERPLASIA
}

\author{
Goncharenko V. M.
}

Doctor of Medical Sciences, Professor, Professor of the Department of Obstetrics and Gynecology № 3

Bogomolets National Medical University

Beniuk V. O.

Doctor of Medical Sciences, Professor, Head of the Department of Obstetrics and Gynecology № 3

Bogomolets National Medical University

\section{Kravchenko Yu. V.}

Postgraduate Student at the Department of Obstetrics and Gynecology № 3 Bogomolets National Medical University

Kovaliuk T. V.

Candidate of Medical Sciences, Associate Professor at the Department of Obstetrics and Gynecology № 3 Bogomolets National Medical University

\section{Komar V. M.}

Doctor-Intern at the Department of Obstetrics and Gynecology № 3

Bogomolets National Medical University

Kyiv, Ukraine

In recent years, there has been an increase in the incidence of endometrial hyperplasia in most countries of the world, including Ukraine [1].

Endometrial hyperplasia (EH) is a peculiar form of uterine pathology that reflects a wide range of endometrial morphological disorders, mainly an increase in the gland/stroma ratio compared to normal proliferative endometrium.

The clinical significance of endometrial hyperplasia lies in the associated risk of progression to endometrial cancer, and «atypical» forms of hyperplasia are considered as precancerous diseases [2].

Endometerial cancer (EC) is one of the most common malignant gynecological nosologies in the developed world, especially among menopausal women (according to Cancer research, UK). The risk of developing of endometrial cancer is determined by morphofunctional features of various types of hyperplasia, as well as epigenetic and genetic disorders that lead to inactivation tumor-suppressive genes, increased proliferation, angiogenesis, and 68 
decreased apoptosis $[2,3,4,5]$. In connection with an extreme clinical significance of endometrial hyperplasia, including as a risk factor for the malignant process, there has recently been a trend to study the molecular genetic foundations of the development $\mathrm{EH}$, and the search for predictors of its formation and progression into EC. The results of the conducted studies were unclear, and the implementation of the results in clinical practice was not significantly widespread. In some studies, the following indicators were selected as predictive markers: loss of PTEN expression, mutations of the MSH1 repair gene, a decrease in apoptotic index BCL-2/BAX [6, 7, 8, 9], PAX2 [6], as well as a low level of expression of both progesterone receptor (PG) isoforms. Other studies have identified other indicators: a decrease in COX-2 expression, an increase in P16 expression, and the value of the morphometric indicator D-score $<1$ [10]. In the literature, other molecular genetic markers can be found - MSI, MSH1, KRAS, $\beta$-catenin [10].

Traditional histopathological classification systems for EH demonstrate a wide and different degree of diagnostic reproducibility and, as a result, standardized patient management becomes complex [2]. The most common treatment strategy for EH is progesterone hormonetherapy, but standartized treatment regimens for various histopathic forms of $\mathrm{EH}$, taking into account the expression of specific proteins, have not been developed [1].

Despite the prevalence of EH and EC, insufficient implementation of the study of molecular genetic markers of EH and EC in clinical practice, the lack of a standardized approach to the management and treatment of EH depending on histological and immunohistochemical features, the study of this problem is extremely relevant, both from a scientific and practical point of view.

The aim of the study is to increase the effectiveness of treatment of patients with simple hyperplasia of the endometrium by developing a therapeutic and diagnostic algorithm taking into account the immunohistochemical profile of the hyperplastic endometrium.

Materials and methods. A full range of examinations and treatment was performed according to the data obtained from 203 women of reproductive and premenopausal age with histologically confirmed simple hyperplasia of the endometrium. We have proposed a method of integral assessment of the endometrial condition, which includes a number of immunohistochemical criteria that allow us to assess the sensitivity of the endometrium to hormonal drugs (endometrial receptor phenotype), the state of pro - and antiapoptic mechanisms for maintaining an equilibrium between the processes of proliferation and apopotosis, the degree of activation of the diffuse endocrine system-APUD-system (concentration ES cells in the endometrium) hyperplastic endometriosisI in this category of patients. Based on the re- 
sults of the study, a method of hormone therapy was developed, proposed and implemented in clinical practice, taking into account the state of the receptor systems and the level of activation of apoptosis, as well as the diffuse endocrine system - APUD system of the hyperplastic endometrium. Patients of reproductive age received didrogesterone from day 5 to 25 at a dose of $20 \mathrm{mg}$ per day, patients of perimenopausal age have received medroxyprogesterone acetate $300 \mathrm{mg}$ per week. The criterion for choosing hormone therapy with these drugs is the presence of progesterone receptor expression at least 100 points on the HISTOScore scale. While detecting progesterone receptor expression below 100 points (low sensitivity), triptorelin was prescribed for 3 months. Control of the effectiveness of hormonal treatment was performed by ultrasound examination of the pelvic organs after 3 and 6 months of treatment and by separate diagnostic curettage of the uterine mucosa and the cervical canal under the control of a hysteroscope.

A control group was formed of 96 women with simple endometrial hyperplasia who underwent hormone therapy without taking into account immunohistochemical data.

The results of the study. Analysis of the obtained data showed that in the group of women with simple endometrial hyperplasia without atypia in the reproductive age of $13.1 \%$ and premenopausal patients in $18.5 \%$ of patients progestin-insensitive form of the pathological process was observed.

An analysis of the effectiveness of individualized treatment of patients of the reproductive age with simple EH who received progestins as hormone treatment for 6 months, showed that in the group with personalized treatment, the effectiveness was $24.0 \%$ higher than in the group, where the treatment was performed without determining the receptor state of endometrium. A similar trend was observed in the group жінок of premenopausal women with simple EH : in the group with personalized treatment, complete morphological effect was observed in $81.5 \%$ of patients, which exceeds the effectiveness of treatment by $20.6 \%$ compared to the group with standard treatment, where this indicator was $60.9 \%$. The prescription of GnRH agonists for menostasis within 6 months leads to the restoration of endometrial sensitivity to progestins, the establishment of physiological ratios of the receptor systems and the balance between the processes of apoptosis and proliferation, which allows to perform pathogenetically directed, step-by-step rational therapy of endometrial hyperplastic processes in women of reproductive and premenopausal age.

Conclusions. The main criterion of endometrial sensitivity to progestin therapy is the presence of a sufficient concentration of progesterone - PR 
receptors and the preservation of the ratio with estrogen receptors ER - the physiological ratio ER/PR is equal to 1 .

The individualized approach to the treatment of patients with simple endometrial hyperplasia, taking into account the indicators of its immunohistochemical profile, allows to optimize treatment, avoid relapse, improve the results of therapy in the reproductive age by $24.0 \%$, in premenopause by $20.6 \%$, and serves as a qualitative prevention of endometrial cancer in the future.

\section{References:}

1. Benyuk V.O. Modern determinants of endometrium hyperplastic processes pathogenesis / Benyuk V.O., Goncharenko V. M., Nykoniuk T. R. // Health of Women. 2016.5(111):137-142; doi 10.15574/HW. 2016.111.137

2. P. A. Sanderson. New concepts for an old problem: the diagnosis of endometrial hyperplasia./ P. A. Sanderson, H. O. D. Critchley, A. R. W. Williams, M. J. Arends, P.T.K. Saunders // Hum Reprod Updat.2017 1;23(2):232-254. doi: 10.1093/humupd/dmw042.

3. Bishtawi A. H. To the question of pathogenesis of endometrial hyperplasia. / Bishtawi A. H. [ and others ] // Tumors of the female reproductive system. 2012, no. 3-4, Pp. 108-112.

4. Wheeler, D. T. Histologic Alterations in Endometrial Hyperplasia and Well-differentiated Carcinoma Treated With Progestins / D. T. Wheeler, R. E. Bristow, R. J. Kurman // Am. J. Surgical Pathology. 2007. Vol. 31 (7). P. 208-231.

5. Yang, S. Progesterone: the ultimate endometrial tumor suppressor / S. Yang, K. W. Thiel, K. K. Leslie // Trends Endocrinol. Metab. 2011. Vol. 22 (4).

6. A. Raffone. PAX2 in endometrial carcinogenesis and in differential diagnosis of endometrial hyperplasia: A systematic review and metaanalysis of diagnostic accuracy./ A. Raffone, A. Travaglino, G. Saccone, M. Mascolo, L. Insabato, A. Mollo, G. De Placido, F. Zullo. //Acta Obstet Gynecol Scand 2019;98(3):287-299.

7. A. Raffone. PTEN expression in endometrial hyperplasia and risk of cancer: a systematic review and meta-analysis./ A. Raffone, A. Travaglino, G. Saccone, M. Viggiani, P. Giampaolino, L. Insabato, A. Mollo, G. De Placido, F. Zullo// Arch.Gynecol Obstet 2019;299(6):1511-1524

8. H. Wu. Expression of MMR in endometrial adenocarcinoma in women under 50 years old./ H. Wu., J. Song, Yi. Shi,Yi. Liu// Zhonghua Bing Li Xue Za Zhi 2012;41(11):733-6. 
9. S. M. Samani. Expression of p53, Bcl-2 и Bax in endometrial carcinoma, endometrial hyperplasia and normal endometrium: a study histopathological / S. M. Samani., T. E. Bojnordi, M. Zarghampour, S. Merat, D. F Fouladi // J. Obstet Gynaecol. 2018;38(7):999-1004.

10. A. Steinbakk. Molecular biomarkers in endometrial hyperplasias predict cancer progression / A. Steinbakk [ et al. ] // Am. J. Obstet. Gynecol. 2011. Vol. 204 (4), P. 450-454

11. M. H. Auclair. Guideline № 392-Classification and Management of Endometrial Hyperplasia/ M. H. Auclair, P. J Yong, Sh. Salvador, J. Thurston, T.T.J Colgan, Alexandra Sebastianelli J. // Obstet Gynaecol Can 2019

DOI https://doi.org/10.30525/978-9934-588-81-5-1.19

\section{NEONATAL ARRHYTHMIA: DEVELOPMENT RISK FACTORS}

\section{Gonchar M. O.}

MD, PhD, Doctor of Med. Science, Professor, Head of the Department of Pediatrics № 1 and Neonatology

Kharkiv National Medical University

\section{Ivanova Ye. $\mathrm{V}$.}

$M D$, Assistant at the Department of Pediatrics № 1 and Neonatology

Kharkiv National Medical University

\section{Tokariev V.S.}

5th year Student at the Medical Faculty № 3

Kharkiv National Medical University

\section{Kolesnikov I. R.}

5th year Student at the Medical Faculty № 3

Kharkiv National Medical University

Pizniak H. O.

5th year Student at the Medical Faculty № 3

Kharkiv National Medical University

Kharkiv, Ukraine

Neonatal arrhythmias are considered as manifestations of impaired adaptation of the cardiovascular system in newborns in the postnatal period $[4$, p. 345] and can lead to the development of life-threatening conditions $[2$, p. 384]. The causes of cardiac arrhythmias at this age can be various 